\title{
Evolution of a rapid response team over a ten-year period: One academic center's experience
}

\author{
Amanda Venable MSN
}

$\boldsymbol{R}_{\text {apid }}$ response teams (RRTs) became embedded in US hospitals following the launch of the 100,000 Lives Campaign in 2004 by the Institute for Healthcare Improvement and the introduction of RRTs as one of six initiatives to improve the quality of patient care. The use of RRTs also allows hospitals to meet a Joint Commission requirement to implement a mechanism that enables staff members to obtain help from experts when their patients' conditions are deteriorating. ${ }^{1}$ This article reviews performance data from an Academic Medical Center and recent rapid response literature.

Rapid response teams have been in place in many hospital systems for more than 15 years with the primary objective of reducing avoidable adverse patient events. Although rapid response systems have very different structures in various health systems, the International Society for Rapid Response Systems (iSRRS) describes these systems as having four basic components: ${ }^{2,3}$

1. The afferent limb (information inward)

2. The efferent limb (information outward)

3. Process improvement

4. Governance

\section{INFORMATION COLLECTION (AFFERENT LIMB)}

The afferent limb of RRTs provides a method or means for identifying patients and escalating care when appropriate. The rapid response literature indicates that relying on healthcare staff alone to identify

Corresponding author: Amanda Venable Contact Information: Amanda.venable@ umchealthsystems.com

DOI: 10.12746/swrccc.v7i31.591 deteriorating patients is not necessarily adequate for the afferent limb. The iSSRS recommends the involvement of patients and families in activating rapid response systems. ${ }^{3}$ Most robust systems utilize an Early Warning Score (EWS) to facilitate the afferent limb of their rapid response program. Kramer reported a reduction in codes outside the intensive care unit (ICU), and EWS and proactive rounds have reduced unplanned ICU transfers. ${ }^{4}$ Early warning scores consist of real time, actionable physiologic metrics which trigger event detection and decision points. One example is the Modified Early Warning Score (MEWS) which includes physiologic measures such as: ${ }^{4}$

- Respiratory rate

- Heart rate

- Systolic blood pressure

- Level of consciousness

- Temperature

- Hourly urine output

The afferent limb of the rapid response system used at University Medical Center in Lubbock, Texas, utilizes the St. John Sepsis Surveillance Agent. This program uses real time clinical data uploaded into the cloud within the electronic health record (EHR) to analyze and detect sepsis early. The surveillance agent uses a proprietary algorithm to detect potential sepsis cases so the program differs from using standard Sepsis-related Organ Failure Assessment (SOFA) or Q-SOFA criteria. When detection of a patient's potentially developing systemic inflammatory response syndrome (SIRS) or sepsis occurs, an alert fires and notifies the rapid responder of the event. Input from rapid responders in our organization has concluded the alerts may not be specific to sepsis but do identify patients outside critical care units who may have had a change in clinical status. For this reason, the RRT has adapted the use of the St John 


\begin{tabular}{|c|c|c|c|c|c|c|c|}
\hline & 3 & 2 & 1 & 0 & 1 & 2 & 3 \\
\hline $\begin{array}{l}\text { Respiratory Rate } \\
\text { per minute }\end{array}$ & & $\begin{array}{c}\text { Less than } \\
8\end{array}$ & & $9-14$ & $15-20$ & $21-29$ & $\begin{array}{c}\text { More than } \\
30\end{array}$ \\
\hline $\begin{array}{l}\text { Heart Rate } \\
\text { per minute }\end{array}$ & & $\begin{array}{l}\text { Less than } \\
\quad 40\end{array}$ & $40-50$ & $51-100$ & $101-110$ & $111-129$ & $\begin{array}{c}\text { More than } \\
129\end{array}$ \\
\hline $\begin{array}{l}\text { Systolic } \\
\text { Blood Pressure }\end{array}$ & $\begin{array}{l}\text { Less than } \\
70\end{array}$ & $71-80$ & $81-100$ & $101-199$ & & $\begin{array}{l}\text { More than } \\
200\end{array}$ & \\
\hline $\begin{array}{l}\text { Conscious } \\
\text { level (AVPU) }\end{array}$ & Unresponsive & $\begin{array}{l}\text { Responds } \\
\text { to Pain }\end{array}$ & $\begin{array}{l}\text { Responds } \\
\text { to Voice }\end{array}$ & Alert & $\begin{array}{l}\text { New agitation } \\
\text { Confusion }\end{array}$ & & \\
\hline $\begin{array}{l}\text { Temperature } \\
\text { ( } \mathrm{C} \text { ) }\end{array}$ & & $\begin{array}{l}\text { Less than } \\
35.0\end{array}$ & $35.1-36$ & $36.1-38$ & $38.1-38.5$ & $\begin{array}{l}\text { More than } \\
38.6\end{array}$ & \\
\hline $\begin{array}{l}\text { Hourly Urine } \\
\text { For } 2 \text { hours }\end{array}$ & $\begin{array}{l}\text { Less than } \\
10 \mathrm{mls} / \mathrm{hr}\end{array}$ & $\begin{array}{l}\text { Less than } \\
30 \mathrm{mls} / \mathrm{hr}\end{array}$ & $\begin{array}{l}\text { Less than } \\
45 \mathrm{mls} / \mathrm{hr}\end{array}$ & & & & \\
\hline
\end{tabular}

Sepsis Surveillance Agent as an EWS rather than a sepsis alert system alone. Through this system, an alert fires and the rapid response nurse opens the patient's EHR to review the medical history, current medications, and vital sign trends. In the event the rapid responder determines the patient may be developing a new clinical condition or has a deterioration in clinical status, the rapid response nurse goes to the patient's bedside to assess the patient and collects additional clinical information. The rapid response nurse collaborates with the primary nurse to determine if additional intervention is needed and more provider involvement is required. It is the rapid responder's responsibility to communicate directly

\section{Result History}

\begin{tabular}{|l|l|l|}
\hline Value & Valid From & Valid Until \\
\hline Systemic inflammatory response syndrome & $10 / 14 / 201916: 40$ CDT & Current \\
\hline
\end{tabular}

\section{Result Comments Action List}

1.) (Medium Importance) Result Comment by SYSTEM on October 14, 2019 16:40 US/Central $7 \mathrm{~d} 3 \mathrm{cb} 678-\mathrm{e} 700-4672-\mathrm{aca} 3-\mathrm{a} 82 \mathrm{f3} 2138 \mathrm{c58} \mid$

10/14/2019 09:32 White Blood Cells (12.54 x10E9/L)|10/14/2019 15:22 RR (25 br/min) |

10/14/2019 15:45 Heart Rate (112 bpm) 
with the primary provider to assure prompt awareness of the patient's change in condition. In the event the patient does not require a transfer to a critical care unit at the time of assessment, the rapid responder will assess the patient again at two, six, and twelve hours post the initial alert. At twelve hours post alert, the rapid responder usually stops following the patient unless additional alerts alarm the RRT. The picture below is an example of a sepsis alert reviewed by the rapid response nurse within the sepsis alert system.

Despite using an early alert system, the detection of patient deterioration can remain suboptimal, leading to the development of potentially avoidable adverse events, such as codes outside the ICU. ${ }^{5}$ The RRT in our hospital has found that suboptimal management of vital signs, both delayed documentation and inaccurate documentation, can create important problems. When vital signs are not documented in real-time, this delays the notification of the RRT about a potentially deteriorating patient and can delay timely intervention.

An additional component of the afferent limb includes an automatic rapid response nurse visit for patients who transfer out of the ICU. When a patient transfers to a lower level of care from an ICU, the rapid response nurse evaluates the patient at two, six, and twelve-hour intervals post ICU transfer. During the patient visit, the nurse assesses the patient's clinical status to monitor for any signs of deterioration, thereby providing an improved transition of care within the healthcare team.

The iSSRS recommends that one of the afferent limbs of RRTs should include patients and family members. Within our organization, the phone number to call the rapid response nurse is provided to all patients. An interesting observation from our RRT notifications has been that RRT calls initiated by patients and /or families average fewer than one call per month, many fewer than originally expected. The most frequent calls initiated by either patients or family stem from a lack of understanding of their treatment plans. Therefore, the RRT serves a unique role and opportunity to facilitate a team based approach to include the patient and family within a personalized plan of care.

A unique component of our hospital's RRT, contributing to both the afferent and efferent limbs, is the addition of vascular access. Rapid responders are trained to place peripherally inserted central catheters, midlines, and ultrasound guided peripheral IVs. This allows the RRT to be more effective with interventions in deteriorating patients with poor IV access and to further identify deteriorating patients through vascular access calls. A common reason for an inability to obtain IV access is often hypovolemia. When rapid responders are called for vascular access, they often identify other issues requiring additional interventions to prevent patient deterioration.

\section{RESPONSES TO NEW INFORMATION (EFFERENT LIMB)}

The efferent limb of a RRT involves the type of clinical response. The majority of rapid response systems respond with a registered nurse (RN); however, multiple variations of team structures exist and could include an Advanced Practitioner RN, physician intensivist, or hospitalist. Our facility uses a RN for initial response. The rapid response nurse then notifies the ICU resident if needed when the initial assessment determines the need for additional orders. While waiting for physician response, the RRT begins to intervene with a deteriorating patient by using the Rapid Response Standing Delegation Orders. These orders cover interventions needed immediately for the diagnosis and treatment of the deteriorating patient. For example, a chest pain patient will have a 12 lead ECG and troponins drawn while waiting on a call back from the physician. Rapid responders will collaborate with the care team and together, with the provider, determine if the patient requires transfer to a higher level of care.

\section{Process improvement}

The process improvement arm of rapid response follows recommended rapid response system metrics. Example of metrics may include, but are not limited to:

- Call volume

- Call volume type (clinical deterioration or IV access)

- Cardiac arrests out of the ICU 
Cardiac arrests outside the ICU serve as a strong indicator of an organization's efforts toward continuous improvement of the RRT and all nursing staff. Any cardiac arrest outside of the ICU is presented during rapid response morbidity and mortality monthly conferences during which rapid responders and medical-surgical nursing staff are invited to review the case and identify opportunities for improvement. The patient's case is presented and assessed to determine if clinical deterioration was recognized and acted upon in a timely manner. In the event opportunities for improvement are identified, the findings are acted upon through the governance arm of the RRT.

\section{TEAm MANAGEMENT (GOVERNANCE)}

The governance arm of the RRT is responsible for the daily management and improvement of the team. The governance arm consists of a critical care Nursing Director and RRT members. The Nurse Director has responsibility of the RRT and reports findings to the hospital wide safety committee and the Critical Care Collaborative, an interprofessional team from all ICUs and medical providers.

\section{Discussion}

The RRT at this academic medical center was created in 2007. As the RRT developed over time, various metrics were identified and monitored for improvement. The organization saw a continuous decrease in number of codes outside the ICU from 2011 until 2017. Changes within the organization during 2018 included census growth and a need for a Medical-Surgical Intermediate Care Unit. Adding the higher acuity floor in an area away from the other ICUs has created challenges and increased the number of cardiac arrests outside the ICU. To mitigate this risk, rapid responders have recently provided education to the non-ICU units about recognizing critical illness, including education regarding recognizing abnormal vital signs for non-licensed personnel. Related to the observed increase, rapid responders began regular rounding with patients who transferred out of the ICU for the first 12-hours post transfer.

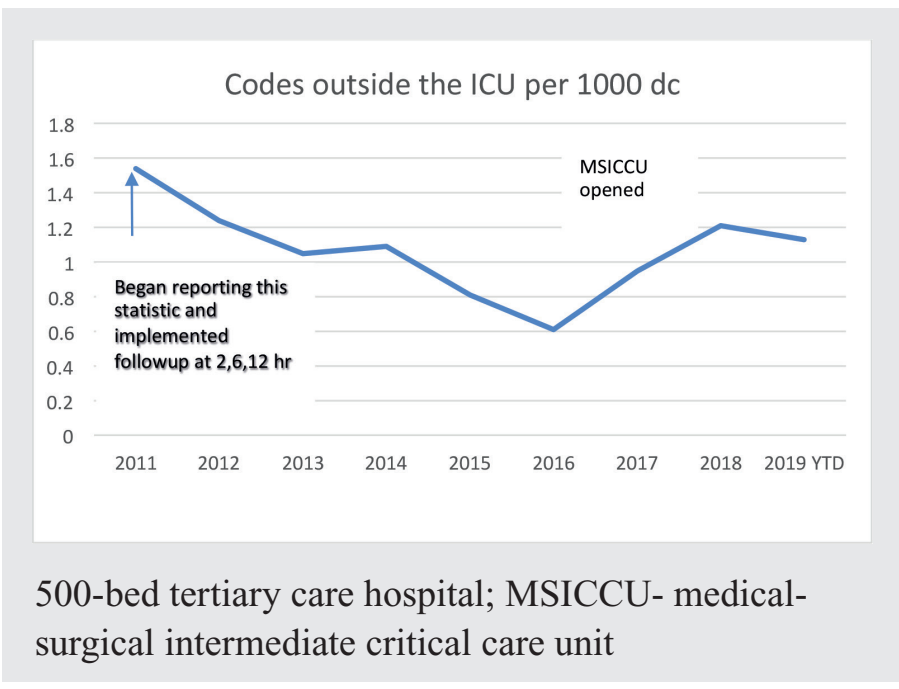

As RRTs continue to evolve, the challenge will be to intervene in the course of a patient's care in a meaningful way that decreases mortality. According to White et al, $19 \%$ of patients activated for rapid response die within 28 days. ${ }^{6}$ Following the highest risk patients through rapid response systems creates a heavy workload and burden on RRTs. In our hospital RRT calls per 1000 discharges have gone up significantly as the effort to identify high risk patients has increased. The increase in this activity is also directly linked to the activation of the St John sepsis screening activity. Due to the increase in workload of the RRT, additional full-time equivalents have been added so the organization has two rapid responders working each shift. The RTT sees approximately 150 deteriorating patients, 350 patients for vascular access, and 700 patients for

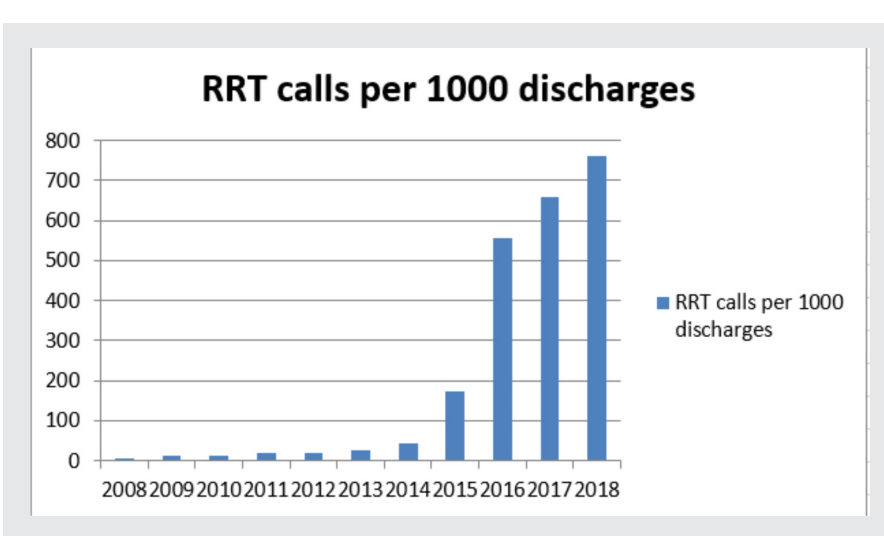


sepsis screening per month. The remaining calls are responses to other clinical questions or requests for assistance.

A potential benefit of RRT which often is underreported is the support clinical nurses have when the team is assisting with complex patients. Further, having an RRT provides a continuous educational opportunity for nurses outside of the critical care environment. Nurses appreciate the assistance with vascular access and frequently have inadequate resources for patients needing additional support. One nurse from a medical-surgical area stated "Rapid response assists greatly with critical patients, collaborating with the multidisciplinary team to get the patient to the appropriate level of care. They are very informative and give great patient education." Nurses outside the ICUs also value the support of having an experienced nurse facilitate and monitor trended vital signs over time.

Looking forward to the future of rapid response teams, an accurate predictive model to determine the patients requiring close RRT follow-up to improve mortality is important future research. A predictive model is key to allowing rapid responders, with limited resources, to focus on patients with higher acuity on whom they can have the highest clinical impact. As more chronically ill patients are hospitalized, it is imperative to determine how to decide who is at risk of cardiac arrest and requires a higher level of care. Rapid response teams have a critical opportunity to improve patient outcomes by reducing mortality and assuring patients are placed in the appropriate level of care setting.

Keywords: rapid response team, cardiac arrest, early warning system, sepsis
Article citation: Venable A. Evolution of a rapid response team over a ten-year period: one academic center's experience. The Southwest Respiratory and Critical Care Chronicles 2019;7(31): 8-12

From: Trauma and Surgical ICU, Burn Center, Rapid Response Team, University Medical Center, Lubbock, Texas

Submitted: 9/27/2019

Accepted: 10/16/2019

Reviewer: Drew Payne DO

Conflicts of interest: none

This work is licensed under a Creative Commons Attribution-ShareAlike 4.0 International License.

\section{REFERENCES}

1. Stolldorf D, Jones C. The deployment of rapid response teams in U.S. hospitals. Jt Comm J Qual Patient Saf 2015;41(4): 186-183.

2. Society for Rapid Response Systems. https://rapidresponsesystems.org/

3. Subbe C, Bannard-Smith J, Bunch J, et al. Quality metrics for the evaluation of Rapid Response Systems: Proceedings from the Third International Consensus Conference on Rapid Response Systems. Resuscitation 2019;141:1-12.

4. Kramer A, Sebat F, Lissauer M. A review of early warning systems for prompt detection of patients at risk for clinical decline. J Trauma Acute Care Surg 2019;87:1, S67-S73.

5. Wood C, Chaboyer W, Carr P. How do nurses use early warning scoring systems to detect and act on patient deterioration to ensure patient safety? A scoping review. Int J Nurs Study 2019 Jun;94:166-178.

6. White K, Bernard A, Scott I. Derivation and validation of a risk score for predicting mortality among inpatients following rapid response team activation. Postgrad Med J 2019 Jun;95(1124):300-306. 